\title{
FIRST OBSERVATIONS OF AN R CORONAE BOREALIS STAR WITH THE SPACE TELESCOPE IMAGING SPECTROGRAPH: RY SAGITTARII NEAR MAXIMUM LIGHT ${ }^{1}$ Geoffrey C. Clayton, ${ }^{2}$ T. R. Ayres, ${ }^{3}$ Warrick A. Lawson, ${ }^{4}$ John S. Drilling, ${ }^{2}$ P. WOITKe, ${ }^{5}$ AND MARTIN ASPLUND ${ }^{6}$ \\ Received 1998 October 8 ; accepted 1998 November 18
} ABSTRACT

We describe the far-UV (1140-1740 ̊) spectrum of the hydrogen-deficient R Coronae Borealis (RCB) star RY Sgr, obtained near maximum light (pulsational phase $\sim 0.1$ ) by the Space Telescope Imaging Spectrograph (STIS) on Hubble Space Telescope. The far-UV spectrum shows a photospheric continuum rising steeply toward longer wavelengths and two prominent emission features at the shorter wavelengths: C II $\lambda 1335$ and $\mathrm{Cl}$ I $\lambda 1351$ (the latter is radiatively fluoresced by the 10 times stronger $\mathrm{C}$ II multiplet). We also find evidence for $\mathrm{CO} A-X$ 4th-positive system absorption band heads and possible weak CO fluorescent emissions pumped by $\mathrm{C}$ II $\lambda 1335$, but the inferred column densities are low ( $\sim$ few times $\left.10^{16} \mathrm{~cm}^{-2}\right)$, consistent with formation in a warm $(\sim 5000 \mathrm{~K})$ atmospheric layer. The detection of CO molecules, if confirmed, would be significant, because they are thought to play a key role in the dust ejection episodes of RCB stars through the initiation of "molecular cooling catastrophes."

Subject headings: circumstellar matter — stars: individual (RY Sagittarii) stars: variables: other (R Coronae Borealis) — ultraviolet: stars

\section{INTRODUCTION}

The R Coronae Borealis (RCB) stars are a small group of hydrogen-deficient, carbon-rich supergiants that undergo spectacular declines ( $\Delta V$ up to 8 magnitudes) at irregular intervals apparently tied to their pulsation cycles (Clayton 1996). A cloud of carbon-rich dust forms, temporarily eclipses the photosphere (revealing a rich "chromospheric" emission spectrum), and then disperses, allowing the star to return to maximum light. The dust forms in a patch or puff, not as a complete shell. Even if clouds form during every pulsation cycle, only when the dust condenses in the line of sight will a deep decline occur. Thus, the events are irregular and unpredictable. Many of the emission lines seen around RCB stars seem to arise in a region with $T \lesssim 6000 \mathrm{~K}$ and $n_{e} \lesssim 10^{9} \mathrm{~cm}^{-3}$ (Clayton et al. 1992b). Some observed features, such as C II $\lambda 1335$ and C III] $\lambda 1909$, must arise in higher temperature regions.

Extensive ground- and space-based observations of $\mathrm{R}$ $\mathrm{CrB}$, RY Sgr, and V854 Cen have pointed to a possible model of the RCB decline (Clayton et al. 1992a; Whitney, Balm, \& Clayton 1993; Clayton 1996). An apparent connection between pulsational phase and the time of dust formation-seen in RY Sgr and V854 Cen-implies that condensation occurs near the star (Pugach 1977; Lawson et al. 1992). Observed timescales for radiative acceleration of the dust, eclipse of the chromospheric region, and dispersal of the dust add further support to the "near star" model. The main drawback is that dust apparently is condensing

\footnotetext{
${ }^{1}$ Based on observations obtained with the NASA/ESA Hubble Space Telescope, which is operated by STScI for the Association of Universities for Research in Astronomy Inc. under NASA contract NAS 5-26555.

${ }^{2}$ Department of Physics and Astronomy, Louisiana State University, Baton Rouge, LA 70803; drilling, gclayton@rouge.phys.lsu.edu.

${ }^{3}$ CASA, University of Colorado, Boulder, CO, 80309; ayres@vulcan.colorado.edu.

${ }^{4}$ School of Physics, University College, UNSW, Australian Defence Force Academy, Canberra, ACT 2600 Australia; wal@ph.adfa.edu.au.

5 Institut für Astronomie, TU Berlin, Sekr. PN 8-1, Hardenbergstraße 36, D-10623 Berlin, Germany; woitke@physik.TU-Berlin.DE.

${ }^{6}$ NORDITA, Blegdamsvej 17, DK-2100 Copenhagen Ø, Denmark; martin@nordita.dk.
}

within a few stellar radii, or less, of photospheres with $T_{\text {eff }} \sim 5000-7000 \mathrm{~K}$, where equilibrium temperatures should be too hot for nucleation to occur.

The conditions in RCB stars differ in several respects, however, from those occurring in the classical outflows of red giants (Goeres \& Sedlmayr 1992; Whitney et al. 1993; Clayton et al. 1995). First, in RCB stars the mass loss seems to be localized in particular areas of the surface; indeed, there is some evidence that the mass loss might be bipolar (Clayton et al. 1997). In contrast, red giant mass loss is thought to occur more uniformly over the surface, leading to symmetric formation of dust. Second, in RCB stars helium is the dominant buffer gas and hydrogen is only a minority constituent (Lambert 1986); in red giants, carbon nucleation proceeds under hydrogen-rich conditions: the condensation temperature of carbon in the absence of hydrogen is significantly warmer than it is in the presence of hydrocarbons (Donn 1967; Goeres \& Sedlmayr 1992; Cherchneff, Barker, \& Tielens 1992). Third, a large amount of condensable carbon is present in RCB stars, 2 orders of magnitude higher than in red giants. Fourth, at least one RCB star, RY Sgr, has strong shocks propagating through its outer atmosphere, imposing local density enhancements and encouraging nonequilibrium conditions.

The carbon chemistry under these conditions has been described by Goeres \& Sedlmayr (1992) and Woitke, Goeres, \& Sedlmayr (1996). As soon as polar molecules become abundant in the gas, they begin to dominate radiative heating and cooling. At high temperatures, $\mathrm{CO}$ should play an overwhelming role because it is expected to be the most abundant polar molecule by 2 orders of magnitude (Woitke, Goeres, \& Sedylmayr 1996). The CO cools very efficiently, and temperatures in the gas may drop to below $2000 \mathrm{~K}$ (Woitke 1998, private communication). Dust then can form from carbon chains and clusters. Once formed, the dust can be self-shielding. Alternatively, during the passage of a shock, a fluid element first is heated and compressed, then expands, cooling adiabatically. Such shocks have been observed in the atmosphere of RY Sgr (Lawson, Cottrell, \& Clark 1991). When the effects of non-LTE radiative heating and cooling via free-free, bound-free, and line transitions 


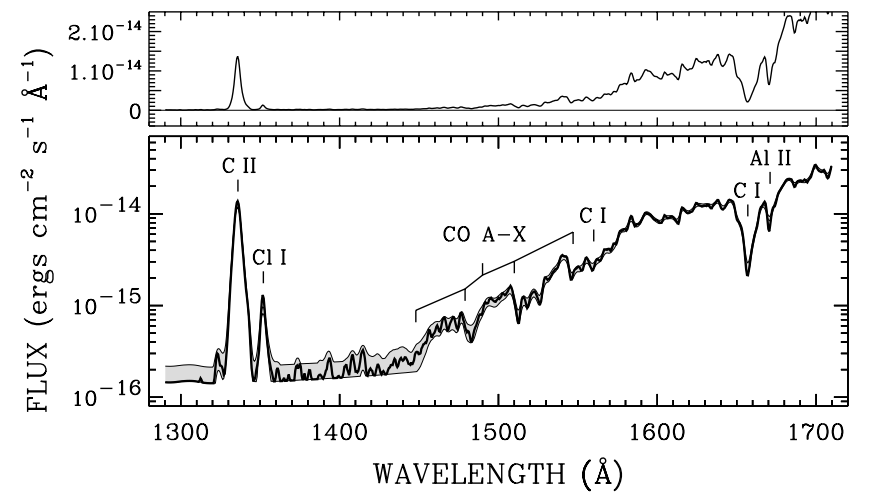

FIG. 1.-Far-ultraviolet spectrum of RY Sgr

(both atoms and molecules) are taken into account, the preconditions for carbon nucleation may be present temporarily; a "puff" of carbon dust could form and then be ejected by radiation pressure.

The observed timescales for RCB dust formation fit in well with those implied by the carbon chemistry model (Woitke et al. 1996). Whether the primary cooling is done by $\mathrm{CO}$ or by the passage of a shock, large amounts of $\mathrm{CO}$ should be present at the time of dust formation; yet there is no published detection of $\mathrm{CO}$ in an RCB star. Unsuccessful searches for IR vibration-rotation bands of $\mathrm{CO}$ have been made in RCB stars, including R CrB and RY Sgr (Wilson, Schwartz, \& Epstein 1973; Zuckerman et al. 1978; Knapp et al. 1982; Lambert 1986; Zuckerman \& Dyck 1986; Wannier et al. 1990; Rao et al. 1991). It has been reported that the HdC star, HD 182040, shows CO absorption bands in the IR (Lambert 1986). Recently, overtone CO absorption has been detected in at least two RCB stars at $2.2 \mu \mathrm{m}$ (Asplund 1998, private communication).

Thus, we turned our attention to the far-ultraviolet, where the $A-X$ 4th-positive system bands of $\mathrm{CO}$ fall; the UV lines are $\sim 10^{3}$ times stronger than their IR counterparts for the same molecular column. There are two RCB stars with well-defined pulsational cycles linked to the time of dust formation: RY Sgr and V854 Cen (Pugach 1977; Lawson et al. 1992). Here we report our initial observations of RY Sgr.

\section{OBSERVATIONS}

RY Sgr was observed with the Space Telescope Imaging Spectrograph (STIS)/Hubble Space Telescope (HST) on 1998 April 25 (JD 2450929), at $\phi=0.1$ of its 38 day pulsational cycle according to the ephemeris of Lawson \& Cot- trell (1990). We employed the G140L low-resolution mode (FUV-MAMA detector), and the $52^{\prime \prime} \times 0$ ".5 slit, yielding a range of $1140-1740 \AA$ with two-pixel resolution $\sim 2 \AA$. We obtained exposures of $1.97 \mathrm{ks}$ and $2.65 \mathrm{ks}$ on consecutive orbits. We co-added the pipeline-processed tracings, weighting by exposure time.

Figure 1 depicts the STIS spectrum of RY Sgr. We corrected the fluxes for reddening according to the Galactic law of Cardelli, Clayton, \& Mathis (1989) assuming $E(B-V)=0.13$ (Asplund et al. 1997). The top panel has a linear flux scale, the bottom panel is logarithmic. In the logarithmic display, the fluxes are truncated at the $2 \sigma$ photometric noise level, and the shaded band depicts $\pm 1 \sigma$. The spectrum is dominated at the shorter wavelengths by the strong $C$ II multiplet emission at $1335 \AA$, and at longer wavelengths by the steeply rising photospheric continuum. Deep absorptions due to atomic carbon $(1657 \AA)$ and ionized aluminum $(1671 \AA$ ) are clearly visible, and several of the weaker absorption dips below $1600 \AA$ coincide with predicted locations of $\mathrm{CO} A-X$ band heads.

The $\mathrm{O}_{\mathrm{I}} \lambda 1305$ resonance multiplet is usually the brightest feature in spectra of normal giants longward of $\mathrm{H}_{\text {I }} \lambda 1215$ Ly $\alpha$. We find no trace of O I in the STIS spectrum of RY $\mathrm{Sgr}$; this is probably not just because of to the low (relative) oxygen abundance $(\mathrm{O} / \mathrm{C}$ is only $10 \%$ the ratio in a solar mixture), but also because the weak hydrogen spectrum inhibits $\mathrm{Ly} \beta$ pumping of $\mathrm{O} \mathrm{I}$ (Bowen fluorescence), which is known to be crucial in the formation of the $\lambda 1305$ triplet in the Sun and other late-type stars (e.g., Skelton \& Shine 1982; Carlsson \& Judge 1993). The absolute oxygen abundance is near solar (Lambert 1986).

We do find a weak feature that we attribute to the $\mathrm{C}$ III $\lambda 1175$ multiplet; C III] $\lambda 1909$ was seen in earlier IUE spectra (Holm et al. 1987; Clayton et al. 1992a; Clayton 1996). Another weak, but detected, feature occurs at $1323 \AA$, just shortward of $C$ II $\lambda 1335$. It probably is the $C$ II $2 \mathrm{~s} 2 \mathrm{p}^{2}$ ${ }^{2} \mathrm{D}-2 \mathrm{p}^{3}{ }^{2} \mathrm{D}^{\circ}$ multiplet whose lower level terminates in the upper state of the $\lambda 1335$ blend and likely is formed by recombination. There is also a significant feature at $1222 \AA$, at the edge of the region affected by Ly $\alpha$ geocoronal contamination (the pipeline processing appears to have compensated for the diffuse $\mathrm{H}$ I emission successfully). The sharp feature might be a $\mathrm{S}$ I line, possibly radiatively fluoresced by Ly $\alpha$ through a $0 \mathrm{eV}$ member of the same multiplet, but there is no obvious feature in the solar spectrum at that wavelength, nor in stellar spectra obtained with GHRS/HST.

The emission feature just longward of C II $\lambda 1335$ very likely is $\mathrm{Cl}$ I $\lambda 1351$. The line appears prominently in solar (and late-type stellar) spectra and is known to be radiatively fluoresced by C II $\lambda 1335$ (Shine 1983). The pumping process

TABLE 1

LiNe FluX MEASUREMENTS

\begin{tabular}{|c|c|c|c|}
\hline $\begin{array}{l}\lambda_{L} \\
(\AA)\end{array}$ & ID & Terms & $\begin{array}{c}f_{L} \\
\left(10^{-15} \mathrm{ergs} \mathrm{cm}^{-2} \mathrm{~s}^{-1}\right)\end{array}$ \\
\hline $1176.2 \ldots \ldots$ & $\mathrm{C}$ III & $2 \mathrm{~s} 2 \mathrm{p}{ }^{3} \mathrm{P}^{\mathrm{o}}-2 \mathrm{p}^{2}{ }^{3} \mathrm{P}$ & $0.51 \pm 0.13$ \\
\hline $1222.2 \ldots \ldots$ & S I? & $3 s^{2} 3 p^{4}{ }^{3} P-3 s^{2} 3 p^{3}\left({ }^{4} S^{o}\right) 11 s^{3} S^{o}$ & $2.1 \pm 0.2$ \\
\hline $1323.7 \ldots \ldots$ & $\mathrm{C}_{\mathrm{II}}$ & $2 s 2 p^{2}{ }^{2} D-2 p^{3}{ }^{2} D^{o}$ & $0.59 \pm 0.04$ \\
\hline $1335.8 \ldots \ldots$ & $\mathrm{C}$ II & $2 \mathrm{~s}^{2}\left({ }^{1} \mathrm{~S}\right) 2 \mathrm{p}{ }^{2} \mathrm{P}^{\mathrm{o}}-2 \mathrm{~s} 2 \mathrm{p}^{2}{ }^{2} \mathrm{D}$ & $19.8 \pm 0.10$ \\
\hline $1351.8 \ldots \ldots$ & $\mathrm{Cl}_{\mathrm{I}}$ & $3 s^{2} 3 p^{5}{ }^{2} P^{o}-3 s^{2} 3 p^{4}\left({ }^{3} P\right) 4 s^{2} P$ & $1.5 \pm 0.1$ \\
\hline
\end{tabular}

NotE. $-f_{\mathrm{L}}$ is not corrected for reddening. Uncertainties are $1 \sigma$. 
will be favored in the photosphere of RY Sgr, with its low densities and correspondingly reduced collisional quenching.

We measured the fluxes of prominent features by means of Gaussian fitting. Results are summarized in Table 1. Previously, Holm \& Wu (1982) reported C II $\lambda 1335$ emission in an IUE low-dispersion spectrum (SWP 15089) of RY Sgr at a level of $2 \times 10^{-14} \mathrm{ergs} \mathrm{cm}^{-2} \mathrm{~s}^{-1}$, in good agreement with the flux measured here. The Holm \& Wu observation occurred at phase 0.5 of the RY Sgr pulsation cycle. So there is no evidence from these two observations for large changes in $\mathrm{C}$ II with pulsation phase. $\mathrm{C}$ II also has been detected in IUE spectra of R CrB and V854 Cen (Holm \& Wu 1982; Holm et al. 1987; Clayton 1996). Both of these stars have integrated fluxes in C II of $\sim 8 \times 10^{-14}$ ergs $\mathrm{cm}^{-2} \mathrm{~s}^{-1}$. HD 182040 shows no obvious emission at $\mathrm{C}$ II (Brunner, Clayton, \& Ayres 1998).

\section{DISCUSSION}

The main goal of our program was to detect carbon monoxide. The far-UV spectra were obtained near phase zero of the RY Sgr pulsational cycle, where dust formation is thought to occur. (There is no evidence from the light curve of RY Sgr, however, that any dust formation did occur during this cycle [Jones 1998, private communication].) The far-UV provides two possibilities: (1) direct absorption of the background photospheric continuum by $\mathrm{CO} A-X$ 4th-positive system bands and (2) fluorescent pumping of $\mathrm{CO}$ by "chromospheric" emissions like C II. There are a number of weak absorption features in the observed spectrum that might satisfy option (1) and several faint emission bumps in the 1370-1420 A interval that might satisfy option (2). We therefore conducted a series of spectral simulations to test these possibilities.

Our results are summarized in Figure 2. Figure $2 a$ compares the observed far-ultraviolet spectrum of RY Sgr (heavy dark curve on the $\pm 1 \sigma$ light-shaded background) with the Asplund et al. (1997) line-blanketed, radiativeequilibrium energy distribution (medium-shaded thick curve), normalized to the mid-UV flux distribution as observed by $I U E$ and smoothed to the $\sim 2 \AA$ resolution

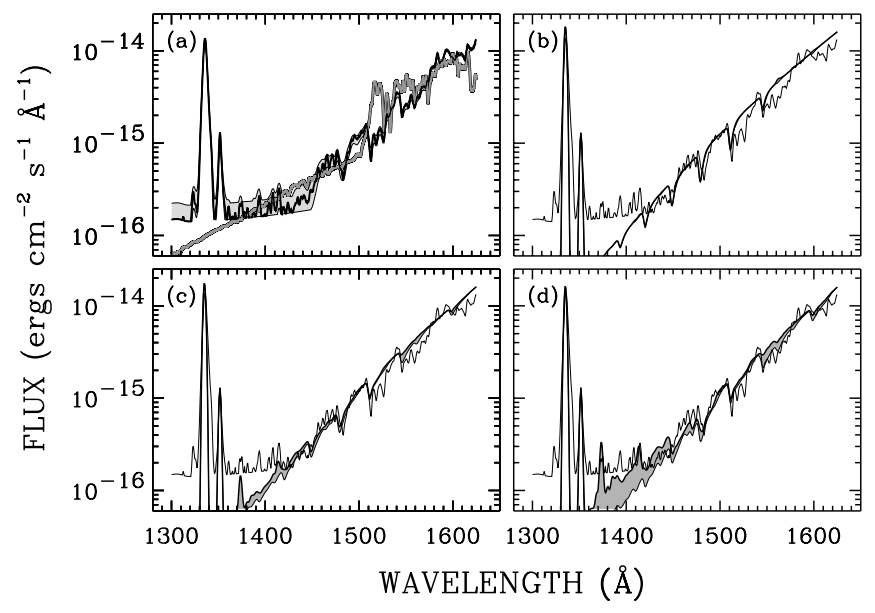

FIG. 2.-(a) Comparison of observed far-ultraviolet spectrum of RY Sgr (dark curve) and Asplund et al. (1997) line-blanketed RE energy distribution (thick medium-shaded curve). (b) Simulated spectrum for $T=1000 \mathrm{~K}$, $N_{\mathrm{CO}}=0.7 \times 10^{16} \mathrm{~cm}^{-2}$, and line FWHM $=10 \mathrm{~km} \mathrm{~s}^{-1}$, representing a cool atmospheric layer. (c) calculation for $\left[3000 \mathrm{~K}, 2 \times 10^{16} \mathrm{~cm}^{-2}, 10 \mathrm{~km}\right.$ $\left.\mathrm{s}^{-1}\right]$. (d) Calculation for [5000 K, $\left.5 \times 10^{16} \mathrm{w} \mathrm{cm}^{-2}, 10 \mathrm{~km} \mathrm{~s}^{-1}\right]$.
(FWHM) of the STIS tracing. The Asplund model matches the ultraviolet energy distribution of RY Sgr remarkably well down to the far-UV interval. There the predicted fluxes are very sensitive to the treatment of line blanketing and to the boundary temperature stratification.

In order to simulate the $\mathrm{CO}$ absorptions, we adopted a simple steeply sloping featureless continuum with superimposed Gaussian emission lines representing the $\mathrm{C}$ II $\lambda 1335$ multiplet and $\mathrm{Cl}$ I $\lambda 1351$. We adopted line widths of $\mathrm{FWHM}=25 \mathrm{~km} \mathrm{~s}^{-1}$, consistent with the "narrow line" region of the chromosphere of RY Sgr (Lambert, Rao, \& Giridhar 1990; Asplund 1995).

Figure $2 b$ compares the observed spectrum with a featureless continuum absorbed by $\mathrm{CO} A-X$ bands in a layer of $T=1000 \mathrm{~K}, \quad N_{\mathrm{CO}}=0.7 \times 10^{16} \mathrm{~cm}^{-2}$, and line $\mathrm{FWHM}=10 \mathrm{~km} \mathrm{~s}^{-1}$, appropriate to the turbulent broadening high in the atmosphere of RY Sgr (Asplund et al. 1997). We assumed isotopic ratios of ${ }^{12} \mathrm{C} /{ }^{13} \mathrm{C} \sim 100$ (as observed: Lambert \& Rao 1994; Pollard, Cottrell, \& Lawson 1994), and ${ }^{16} \mathrm{O} /{ }^{18} \mathrm{O} \sim 100$ (Lambert et al. 1998). We used the Kurucz (1976) line lists and the $A-X$ branching ratios given by Durrance (1980). The simulated band shapes are not particularly sensitive to the oxygen isotope ratio, nor to that of carbon, as long as the minority isotope has low abundance (as observed). All of the simulated spectra were smoothed to $2 \AA$ resolution.

Figure $2 c$ depicts an analogous calculation for parameters $3000 \mathrm{~K}, 2 \times 10^{16} \mathrm{~cm}^{-2}$, and $10 \mathrm{~km} \mathrm{~s}^{-1}$. The lower trace indicates the "pure absorption" case: photons absorbed by the CO lines are "destroyed" by collisions. The upper trace (connected to the lower one by shading) represents the "pure scattering" case: all photons absorbed by $\mathrm{CO} A-X$ transitions are reemitted in other $\mathrm{CO} A-X$ lines according to selection rules and branching ratios (e.g., Ayres et al. 1997); photons dropping into high-opacity transitions will continue to scatter until reemitted in lowopacity lines.

Pure scattering fluorescence likely is the most appropriate situation for the outer atmosphere of RY Sgr. The radiative lifetime of the excited $A{ }^{1} \Pi$ electronic state is only $t_{R} \sim 10^{-7} \mathrm{~s}$. The collisional quenching rate-dominated by the majority constituent helium-is $\sim\langle\sigma v\rangle n_{\mathrm{He}}$ (e.g., Durrance 1980), where the hard-sphere cross section is $\sigma \sim$ few times $10^{-17} \mathrm{~cm}^{2}$, the mean thermal velocity of helium atoms is $v \sim 5 \times 10^{5} \mathrm{~cm} \mathrm{~s}^{-1}(T=5000 \mathrm{~K})$, and the helium number density high in the atmosphere of RY Sgr is $n_{\mathrm{He}} \sim$ $10^{12} \mathrm{~cm}^{-3}$ (based on density arguments developed below, and with $\mathrm{He} / \mathrm{C} \sim 100$ ). The inverse of the quenching rate yields a collisional "survival time" of $\sim 10^{-1} \mathrm{~s}$, considerably longer than $t_{R}$. Thus, photons can scatter many times through $A-X$ transitions before being thermalized by a collision. The main limitation on repeated scatterings will be absorptions in the far-UV continuum.

Figure $2 d$ is similar to Figure $2 c$, but for $5000 \mathrm{~K}, 5 \times 10^{16}$ $\mathrm{cm}^{-2}$, and $10 \mathrm{~km} \mathrm{~s}^{-1}$. In this case, pumping by C II (and $\mathrm{Cl}$ I) is more important, and weak fluorescent emissions appear at $\sim 1371 \AA$ and $\sim 1412 \AA$. In Figures $2 b-2 d$ we selected $N_{\text {Co }}$ for each temperature scenario to achieve a good match between the simulation and the observed spectrum. A change in the column density by a factor of 2 would noticeably worsen the agreement in each case.

In the low-temperature simulation, only the ground vibrational level $(V=0)$ of the $X^{1} \Sigma$ electronic complex is significantly populated and capable of absorbing the 
far-UV light. Many of the lines are saturated (optically thick) and the frequency-integrated absorption is very sensitive to the Doppler width. As the temperature is raised, for fixed column density, the higher vibrational levels of the ground electronic state become collisionally populated, more of the $A-X$ bands become accessible, and the absorption structure becomes richer. At the same time, the strong lines at low temperature weaken and desaturate. Thus the $3000 \mathrm{~K}$ model with $10 \mathrm{~km} \mathrm{~s}^{-1}$ wide lines predicts a very similar absorption spectrum as the $1000 \mathrm{~K}$ model but requires 3 times the $\mathrm{CO}$ column (and has more fluorescent "filling" in the no-quenching limit). A $1000 \mathrm{~K}$ situation, but with narrow lines (FWHM $\sim$ few $\mathrm{km} \mathrm{s}^{-1}$ ) compatible with a circumstellar site, would show weaker absorption, however, owing to the decreased equivalent width of the saturated lines. In the $5000 \mathrm{~K}$ case, higher vibrational bands lying under the $\mathrm{C}$ II (and $\mathrm{Cl}$ I) lines begin to pump discrete features, much like the situation of O I $\lambda 1305$ fluorescence of CO in red giants (Ayres, Moos, \& Linsky 1983), adding an additional constraint on the molecular column density. We conclude that the possible weak absorption structure and possible weak emission features are consistent with $\mathrm{CO}$ column densities of not more than a few times $10^{16} \mathrm{~cm}^{-2}$, regardless of the temperature of the absorbing "layer." We cannot, however, unambiguously determine the temperature itself or whether the absorption site is atmospheric or circumstellar from the available spectra alone (higher resolution would tell us immediately whether "cool" $[V=0]$ or "hot" $[V>0]$ bands were populated). On the other hand, if the $\mathrm{CO}$ is atmospheric, we can estimate the column that should be present in RY Sgr based on, say, chemical equilibrium, and thereby develop a complementary constraint on the temperature.

Suppose that the $\mathrm{CO}$ forms in an isothermal layer above the photosphere. The accessible molecular absorption column will be limited by the "optical horizon" provided by the background far-UV continuum opacity at those wavelengths. The primary source will be the ground-state photoionization of atomic silicon, with its edge at about $1540 \AA$. Because the density is low, the $\mathrm{Si}^{-\mathrm{Si}^{+}}$balance will be controlled by photoionizations. If we approximate the cross-section as hydrogenic (i.e., $\sim \lambda^{3}$ ) and represent the photospheric radiation field as a diluted Planck function $\left(J_{\lambda} \sim W B_{\lambda}\left(T_{\text {rad }}\right)\right.$, where the dilution factor $\left.W \sim \frac{1}{2}\right)$, then the ionization equilibrium can be written (e.g., Mihalas 1978) as

$$
n_{\mathrm{Si}}=\phi_{\mathrm{Si}}^{-1} n_{\mathrm{e}} \tilde{\epsilon}_{\mathrm{Si}} n_{\mathrm{C}},
$$

where $\tilde{\epsilon}_{\mathrm{Si}} \sim 0.025$ is the $\mathrm{Si} / \mathrm{C}$ abundance ratio and

$$
\phi_{\mathrm{Si}}=W\left(\frac{2 g_{0}^{+}}{g_{0}}\right)\left(\frac{2 \pi m k}{h^{2}} T_{\mathrm{rad}}\right)^{3 / 2}\left(\frac{T}{T_{\mathrm{rad}}}\right)^{1 / 2} e^{-\chi_{\mathrm{I}} / k T_{\mathrm{rad}}}
$$

is the modified "Saha factor."

For $T<6000 \mathrm{~K}$, where the ionization of carbon is minor, we can replace the electron density with $0.03 n_{\mathrm{C}}$, the combined abundance of the easily ionized metals $\mathrm{Si}, \mathrm{Mg}$, and $\mathrm{Fe}$ (Asplund et al. 1997).

We now rewrite equation (1) in terms of column densities by replacing, say, $n_{\mathrm{Si}}$ with $N_{\mathrm{Si}} / H_{p}$, where

$$
H_{p} \sim 2 \times 10^{10}(T / 5000) \mathrm{cm}
$$

is the pressure scale height for a $\log g=0.75$ (e.g., Asplund et al. 1997) helium-rich atmosphere. We thus obtain

$$
N_{\mathrm{Si}}=f\left(T, T_{\mathrm{rad}}\right) N_{\mathrm{C}}^{2} \mathrm{~cm}^{-2} .
$$

The optical depth at the head of the Si continuum is

$$
\tau_{\mathrm{Si}}=\alpha_{\mathrm{Si}} N_{\mathrm{Si}},
$$

where the threshold absorption is $\alpha \sim 7 \times 10^{-17} \mathrm{~cm}^{2}$, based on our hydrogenic fit to cross sections obtained from TOPbase (Cunto et al. 1993). Setting $\tau=1$, we can solve for $N_{\mathrm{C}}$ in terms of $T$ and $T_{\text {rad }}$.

We estimate the $\mathrm{CO}$ column assuming chemical equilibrium formation (the photodissociation threshold of $\mathrm{CO}$ is at $\lambda \sim 1100 \AA$, where the stellar flux is negligible). We write

$$
N_{\mathrm{Co}}=\tilde{\epsilon}_{\mathrm{CO}} N_{\mathrm{C}} \cdot
$$

where $\tilde{\epsilon}_{\mathrm{CO}}$ is the fractional abundance of $\mathrm{CO}$ (relative to carbon)

$$
\tilde{\epsilon}_{\mathrm{CO}}=\frac{\tilde{\epsilon}_{\mathrm{O}} n_{\mathrm{C}} \phi_{\mathrm{CO}}^{-1}}{1+n_{\mathrm{C}} \phi_{\mathrm{CO}}^{-1}} .
$$

Here $\tilde{\epsilon}_{\mathrm{O}} \sim 0.1$ is the fractional abundance of oxygen, and the chemical equilibrium factor $\phi$ (Allen 1973) is

$$
\phi_{\mathrm{CO}}=2.4 \times 10^{25}\left(\frac{T}{5000}\right)^{-0.2} e^{-D_{\mathrm{Co} / k T}} .
$$

In the above, we employed an analytical temperature fit to the $\mathrm{CO}$ partition function.

Given $T$ and $T_{\text {rad }}$ we can calculate the Si-continuumlimited carbon column (and the number density $n_{\mathrm{C}}$ ), from which in turn we can determine $N_{\mathrm{CO}}$. Figure 3 illustrates our results. The thick dark curve is the calculated $\mathrm{CO}$ column for our best guesses of the relevant parameters, particularly the Si I photoionization temperature, $T_{\text {rad }} \sim 4900$ $\mathrm{K}$. We estimated the latter by fitting blackbodies to surface fluxes, derived from the observed energy distribution of RY Sgr, for an assumed stellar radius of $70 R_{\odot}$ and a distance of $2 \mathrm{kpc}$. The light-shaded area depicts the effect of changing the assumed Si I photoionization temperature by $\pm 400 \mathrm{~K}$, equivalent to a factor of $\sim 2$ uncertainty in the stellar angular diameter (a factor of $\sim 4$ in the surface flux). Dashed lines show the impact of a factor of 2 change in the $\mathrm{Si} / \mathrm{C}$ abundance ratio.

At $T<4000 \mathrm{~K}$, the carbon monoxide has fully associated the available oxygen, and $N_{\mathrm{Co}}$ thus is directly proportional to $N_{\mathrm{C}}$. The latter is dictated by $N_{\mathrm{Si}}$ through the $\tau_{\mathrm{Si}}=1$ condition, and the silicon density in turn only weakly

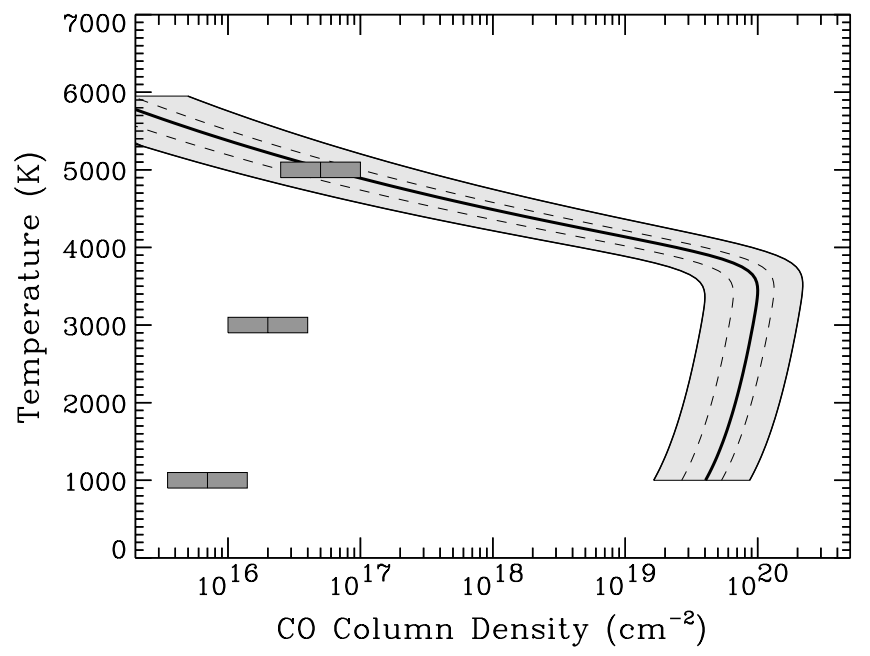

FIG. 3.-Estimated and predicted CO column densities 
depends on temperature, because it is photoionization dominated. Above $4000 \mathrm{~K}$, however, the $\mathrm{CO}$ abundance "desaturates" and $\tilde{\epsilon}_{\mathrm{CO}}$ becomes highly sensitive to temperature (owing to the large dissociation potential, $D_{\mathrm{CO}}=$ $11.09 \mathrm{eV})$.

The narrow dark boxes in Figure 3 are the $\mathrm{CO}$ column estimates obtained from the simulations in Figure $2 b-2 d$. The comparison shows that significant low-temperature regions in the outer atmosphere of RY Sgr at the particular epoch of observation are unlikely. Perhaps that is not surprising, because there was not a subsequent deep minimum. At the same time, the diagram emphasizes that a small change in the thermal structure of the outer atmosphere, say due to postshock cooling, could lead to a tremendous increase in the $\mathrm{CO}$ column density. A 2 order of magnitude increase, corresponding to a few hundred $\mathrm{K}$ temperature drop, would thicken the infrared $\Delta v=1$ (fundamental) bands (near $5 \mu \mathrm{m}$ ) sufficiently to permit efficient surface radiative cooling, and perhaps initiate runaway thermal collapse such as envisioned in the (solar) "thermalbifurcation" mechanism (Ayres 1981; Muchmore \&
Ulmschneider 1985). After all, something must create the chilly conditions suitable for grain formation during RY Sgr's intermittent deep-minimum episodes.

We thus conclude that the weak $A-X$ absorption, which possibly is present in the far-UV spectrum of RY Sgr, probably is characteristic of the normal atmospheric burden of $\mathrm{CO}$ outside of dust formation events. RY Sgr, like other RCB stars, has declines at irregular intervals averaging about 3 yr. There were major declines of RY Sgr in 1990-91 and 1993-95. An observation of the CO bands during such an episode would immediately indicate whether the molecules truly are important in catalyzing the production of the dust clouds.

This work was supported by STScI grant GO-07477.0196A. T. R. A. was supported by grant NAG 5-3226 from NASA to the University of Colorado. We would like to thank Denise Taylor, Steve Hulbert, and Brian Espey for their help in obtaining and reducing the data reported in this paper. We are grateful to Albert Jones for providing brightness estimates of RY Sgr.
Allen, C. W. 1973, Astrophysical Quantities, (London: Athlone)

Asplund, M. 1995, A\&A, 294, 763

Asplund, M., Gustafsson, B., Kiselman, D., \& Eriksson, K. 1997, A\&A, 318, 521

Ayres, T. 1981, ApJ, 244, 1064

Ayres, T. R., Brown, A., Harper, G. M., Bennett, P. D., Linsky, J. L., Carpenter, K. G., \& Robinson, R. D. 1997, ApJ, 491, 876

Ayres, T., Moos, W., \& Linsky, J. 1981, ApJ, 248, L137

Brunner, A., Clayton, G. C, \& Ayres, T. R. 1998, PASP, 110, 1412

Cardelli, J. A., Clayton, G. C., \& Mathis. J. S. 1989, ApJ, 345, 245

Carlsson, M., \& Judge, P. G. 1993, ApJ, 402, 344

Cherchneff, I., Barker, J. R., \& Tielens, A. G. G. M. 1992, ApJ, 401, 269

Clayton, G. C. 1996, PASP, 108, 225

Clayton, G. C.. Kelly, D. M. Lacy, J. H., Little-Marenin, I. R., Feldman, P. A., \& Bernath, P. F. 1995, ÄJ, 109, 2096

Clayton, G. C., Whitney, B. A., Stanford, S. A., \& Drilling, J. S. 1992a, ApJ, 397,652

Clayton, G. C., Whitney, B. A., Stanford, S. A., Drilling, J. S., \& Judge, P. G. 1992b, ApJ, 384, L19

Clayton, G. C., Bjorkman, K. S., Nordsieck, K. H., Zellner, N. E. B., \& Schulte-Ladbeck, R. E. 1997, ApJ, 476, 870

Cunto, W., Mendoza, C., Ochsenbein, F., \& Zeippen, C. J. 1993, A\&A, 275, L5

Donn, B. 1967, in Interstellar Grains, ed. J. M. Greenberg \& T. P. Roark (Washington, DC: USGPO), 191

Durrance, S.T. 1980, Ph.D. thesis

Goeres, A., \& Sedlmayr, E. 1992, A\&A, 265, 216

Holm, A. V., Hecht, J., Wu, C. C., \& Donn, B. 1987, PASP, 99, 497

Holm, A. V., \& Wu, C. C. 1982, in Advances in Ultraviolet Astronomy: Four Years of IUE Research (Washington, DC: USGPO), 429

Knapp, G. R., Phillips, T. G., Leighton, R. B., Lo, K. Y., Wannier, P. G., Wootten, H. A., \& Huggins, P. J. 1982, ApJ, 252, 616

\section{EFERENCES}

Kurucz, R. L. 1976, The Fourth Positive System of Carbon Monoxide, SAO Spec. Rep. 374 (Washington: SAO)

Lambert, D. L. 1986, in Hydrogen Deficient Stars and Related Objects, ed. K. Hunger (Dordrecht: Reidel), 127

Lambert, D. L., \& Rao, N. K. 1994, JApA, 15, 47

Lambert, D. L, Rao, N. K., \& Giridhar, S. 1990, JApA, 11, 475

Lambert, D. L., Rao, N. K., Gustafsson, B., \& Asplund, M. 1998, A\&A, submitted

Lawson, W. A., \& Cottrell, P. L. 1990, MNRAS, 242, 259

Lawson, W. A., Cottrell, P. L., \& Clark, M. 1991, MNRAS, 251, 687

Lawson, W. A., Cottrell, P. L., Gilmore, A. C., \& Kilmartin, P. M. 1992, MNRAS, 256, 339.

Mihalas, D. 1978, Stellar Atmospheres (2d.; San Francisco: Freeman)

Muchmore, D., \& Ulmschneider, P. 1985, A\&A, 142, 393

Pollard, K. R., Cottrell, P. L.,\& Lawson, W. A. 1994, MNRAS, 268, 544

Pugach, A. F. 1977, Inf. Bull. Variable Stars, 1277

Rao, N. K., Nyman, L. A., Nandy, K., \& Houziaux, L. 1991, in The Infrared Spectral Region of Stars, ed. C. Jaschek \& Andrillat, Y. (Cambridge, Cambridge Univ. Press), 361

Shine, R. A. 1983, ApJ, 266, 882

Skelton, D. L., \& Shine, R. A. 1982, ApJ, 259, 869

Wannier, P. G., Sahai, R., Andersson, B.-G., \& Johnson, H. R. 1990, ApJ, 358,251

Whitney, B. A, Balm, S. P., \& Clayton, G.C. 1993, Luminous HighLatitude Stars, ASP Conf. Ser. 45, ed. D. Sasselov, 115

Wilson, W. J., Schwartz, P. R., \& Epstein, E. E. 1973, ApJ, 183, 871

Woitke, P., Goeres, A., \& Sedylmayr, E. 1996, A\&A, 313, 217

Zuckerman, B., \& Dyck, H. M. 1986, ApJ, 304, 394

Zuckerman, B., Palmer, P., Gilra, D.P, Turner, B. E., \& Morris, M. 1978, ApJ, 220, L53 